ARTICLE

Received 17 Dec 2015 | Accepted 4 May 2016 | Published 13 Jun 2016

DOI: $10.1038 /$ ncomms11831

OPEN

\title{
Metal-organic framework with optimally selective xenon adsorption and separation
}

\author{
Debasis Banerjee ${ }^{1}$, Cory M. Simon ${ }^{2}$, Anna M. Plonka ${ }^{3}$, Radha K. Motkuri ${ }^{4}$, Jian Liu ${ }^{4}$, Xianyin Chen ${ }^{5}$, \\ Berend Smit 2,6 , John B. Parise ${ }^{3,5,7}$, Maciej Haranczyk ${ }^{8,9}$ \& Praveen K. Thallapally ${ }^{1}$
}

Nuclear energy is among the most viable alternatives to our current fossil fuel-based energy economy. The mass deployment of nuclear energy as a low-emissions source requires the reprocessing of used nuclear fuel to recover fissile materials and mitigate radioactive waste. A major concern with reprocessing used nuclear fuel is the release of volatile radionuclides such as xenon and krypton that evolve into reprocessing facility off-gas in parts per million concentrations. The existing technology to remove these radioactive noble gases is a costly cryogenic distillation; alternatively, porous materials such as metal-organic frameworks have demonstrated the ability to selectively adsorb xenon and krypton at ambient conditions. Here we carry out a high-throughput computational screening of large databases of metal-organic frameworks and identify SBMOF-1 as the most selective for xenon. We affirm this prediction and report that SBMOF-1 exhibits by far the highest reported xenon adsorption capacity and a remarkable $\mathrm{Xe} / \mathrm{Kr}$ selectivity under conditions pertinent to nuclear fuel reprocessing.

\footnotetext{
${ }^{1}$ Physical and Computational Science Directorate, Pacific Northwest National Laboratory, Richland, Washington 99352, USA. ${ }^{2}$ Department of Chemical and Biochemical Engineering, University of California, Berkley, Berkeley, California 94720, USA. ${ }^{3}$ Department of Geosciences, Stony Brook University, Stony Brook, New York 11794, USA. ${ }^{4}$ Energy and Environmental Directorate, Pacific Northwest National Laboratory, Richland, Washington 99352, USA. ${ }^{5}$ Department of Chemistry, Stony Brook University, Stony Brook, New York 11794, USA. ${ }^{6}$ Institut des Sciences et Ingénierie Chimiques, Valais, Ecole Polytechnique Fédérale de Lausanne (EPFL), Rue de I'Industrie 17, CH-1951 Sion, Switzerland. ${ }^{7}$ Photon Sciences, Brookhaven National Laboratory, Upton, New York 11973, USA.

${ }^{8}$ Computational Research Division, Lawrence Berkeley National Laboratory, Berkeley, California 94720, USA. ${ }^{9}$ IMDEA Materials Institute, C/Eric Kandel 2 , 28906 Getafe, Madrid, Spain. Correspondence and requests for materials should be addressed to B.S. (email: Berend-Smit@berkeley.edu) or to M.H. (email: mharanczyk@lbl.gov) or to P.K.T. (email: Praveen.thallapally@pnnl.gov).
} 
O ne of the grandest challenges of our generation is to meet our rapidly growing energy demand without further increasing the emission of greenhouse gases ${ }^{1,2}$. Nuclear energy is one of the cheapest alternatives to carbon-based fossil fuels that, because of its high energy density and minimal land use requirements, can be scaled up to meet global energy demands. Life cycle analyses indicate that greenhouse gas emissions of a nuclear power plant are significantly lower than fossil fuel technologies and comparable to other renewable electricity generation technologies, such as solar photovoltaics ${ }^{3}$. For the mass implementation of nuclear energy as a lowemissions energy source, we must also safely sequester the associated high-level radioactive waste ${ }^{2}$. In this, most attention is given to recovering the heavy, long-lived nuclear elements in used nuclear fuel (UNF), such as uranium and plutonium. Less discussed are the volatile radionuclides (for example, $\mathrm{Xe}, \mathrm{Kr}$ ) that evolve into the off-gas of UNF aqueous reprocessing facilities ${ }^{4}$. In these off-gases, gaseous radioactive ${ }^{85} \mathrm{Kr}$ has a long half-life $\left(t_{1 / 2}=10.8\right.$ years $)$ and therefore must be captured and removed from the off-gas to prevent its uncontrolled release into the atmosphere 4 . In contrast, the radioactive Xe isotopes $\left(t_{1 / 2} \approx 36.3\right.$ days for ${ }^{127} \mathrm{Xe}$ ) have decayed by the time the fuel is reprocessed. As high purity $\mathrm{Xe}$ is used in many applications, including commercial lighting, propulsion, imaging, anesthesia and insulation, the recovered $\mathrm{Xe}$ could be sold into the chemical market to offset operating costs. At present, cryogenic distillation is the most mature technology to separate Xe and $\mathrm{Kr}$ from air, but it is energy- and capital-intensive and therefore expensive $e^{5,6}$. Furthermore, the radiolytic formation of ozone poses an explosion hazard during cryogenic distillation ${ }^{4}$. These factors incentivize the development of an alternative technology for a less energy-intensive, more cost-effective and safer process to capture $\mathrm{Kr}$ and $\mathrm{Xe}$ from UNF reprocessing facility off-gas.

A promising alternative technology for $\mathrm{Xe} / \mathrm{Kr}$ removal from reprocessing off-gas is an adsorption-based process at room temperature using a selective, solid-state adsorbent. These solidstate adsorbents are found to be almost exclusively Xe-selective, and thus a dual step process whereby, first, the Xe is selectively removed from the off-gas, is a necessary requirement for a practical application ${ }^{7}$. In the subsequent step, the radioactive $\mathrm{Kr}$ can be removed from the Xe-free effluent using the same material or a different material. Adsorbents such as silver-loaded zeolites and activated carbon have been proposed ${ }^{4}$, but these fall short compared with high surface area, crystalline metal-organic frameworks (MOFs) and porous organic cage compounds ${ }^{8-22}$. Among the many novel materials tested thus far, HKUST-1 (ref. 14), Co-formate ${ }^{15,20}$ and CC3 (ref. 12) are shown to be promising for $\mathrm{Xe} / \mathrm{Kr}$ separations, showing high capacity and good selectivity for $\mathrm{Xe}$ over $\mathrm{Kr}$.

An important advantage of MOFs is their chemical tunability; by combining different linkers and metal centres that self-assemble to form ordered, pre-determined crystal structures, one can synthesize millions of possible materials ${ }^{23}$. MOFs can thus be tailor-made to be optimal for applications related to gas storage and separation, catalysis, chemical sensing and optics $9,11,23-34$. Our goal here is to identify an optimal MOF for selectively capturing Xe from the off-gas of UNF reprocessing facilities. In practice, however, constraints in resources allow us to synthesize and test only a small subset of chemical space. Molecular models and simulations of adsorption can rapidly and cost-effectively rank MOFs by their Xe/Kr selectivity with reasonable accuracy (Supplementary Methods). Highthroughput computational screenings thus play a valuable role of elucidating design rules, determining performance limits, and predicting performance rankings of materials to focus experimental efforts on the most promising MOFs for $\mathrm{Xe} / \mathrm{Kr}$ separations ${ }^{35-38}$.

In this work, we use molecular simulations to screen over 125,000 MOF structures ${ }^{39,40}$ for selectively adsorbing Xe over $\mathrm{Kr}$ at dilute conditions pertinent to UNF reprocessing. Our computational screening predicts that one of the most $\mathrm{Xe}$ selective MOFs is a calcium-based nanoporous MOF, SBMOF-1 [also known as CaSDB, $\mathrm{SDB}=4,4$-sulfonyldibenzoate], that has not yet been tested for $\mathrm{Xe} / \mathrm{Kr}$ separations ${ }^{41}$. We affirm this prediction by synthesizing SBMOF-1 and measuring its purecomponent $\mathrm{Xe}$ and $\mathrm{Kr}$ adsorption isotherms. SBMOF-1 exhibits the highest Xe Henry coefficient and thermodynamic $\mathrm{Xe} / \mathrm{Kr}$ selectivity at dilute conditions among MOFs tested to date. In addition to its high thermal and chemical stability, column breakthrough experiments reveal that SBMOF-1 is a practical, near-term material for capturing Xe from reprocessing facilities.

\section{Results}

High-throughput computational screening. For capturing Xe from nuclear reprocessing, the $\mathrm{Xe} / \mathrm{Kr}$ selectivity is the most important thermodynamic property determining the performance of a MOF. We used molecular simulations to predict the $\mathrm{Xe} / \mathrm{Kr}$ selectivity of $125,000 \mathrm{MOF}$ structures at dilute conditions relevant to UNF reprocessing (Supplementary Methods, Fig. 1a,b). The distribution of simulated selectivities in the MOFs is shown in Fig. 1a. We partitioned this distribution into a database of existing MOFs $(\sim 5,000 \text { structures })^{39}$ and a database of predicted/hypothetical structures $(\sim 120,000)^{40}$. These distributions span a large range of selectivities, illustrating the unique tunability of MOF materials. Our simulations predict that the most selective material in the database of existing MOFs is SBMOF-1 (Fig. 1c), a three-dimensional, permanently porous MOF (Cambridge Structural Database (CSD) code: KAXQIL) ${ }^{41}$ Furthermore, the $\mathrm{Xe} / \mathrm{Kr}$ selectivity of SBMOF-1 is ranked in the top 0.01 percentile in the database of 120,000 hypothetical MOFs (Fig. 1b). The red line in Fig. 1b illustrates the outlying $\mathrm{Xe} / \mathrm{Kr}$ selectivity of SBMOF-1 predicted by our screening. While SBMOF-1 has been synthesized and considered for $\mathrm{CO}_{2} / \mathrm{N}_{2}$ separation $^{41}$, it has not been tested for $\mathrm{Xe} / \mathrm{Kr}$ separations.

Synthesis and equilibrium adsorption measurements. Encouraged by the data from our high-throughput screening, we synthesized SBMOF-1 and measured its pure-component Xe and $\mathrm{Kr}$ adsorption isotherms at room temperature (see synthesis section of Supplementary Methods, Supplementary Figs 17-21) ${ }^{41}$. Our first measurement of low pressure Xe uptake in SBMOF-1 at $298 \mathrm{~K}$, when activated by the reported activation procedure ${ }^{41}$, was much lower than predicted by molecular simulation (Supplementary Fig. 22). However, we found that activating SBMOF-1 at a lower temperature yielded low pressure Xe uptake closer to the simulation (Fig. 2a, Supplementary Fig. 23, see effect of activation temperature section of the Supplementary Methods). The Xe adsorption isotherm in SBMOF-1 saturates at a low pressure, indicative of a high affinity for Xe compared with other gases including $\mathrm{Kr}$ (Fig. 2a, Supplementary Figs 24 and 25). The $\mathrm{Kr}$ adsorption isotherm exhibits a smaller slope and does not saturate even at 1 bar, indicative of a much weaker affinity for $\mathrm{Kr}$. This hints that SBMOF-1 is highly discriminatory for $\mathrm{Xe}$ over $\mathrm{Kr}$. Indeed, identifying the Xe and $\mathrm{Kr}$ Henry coefficients from the pure-component adsorption isotherms, we predict SBMOF-1 to exhibit a thermodynamic Xe/Kr selectivity of 16 at dilute conditions at $298 \mathrm{~K}$.

It is interesting to compare the equilibrium $\mathrm{Xe}$ and $\mathrm{Kr}$ uptake of SBMOF-1 with the reported top-performing MOFs. We collected from the literature experimentally measured $\mathrm{Xe}$ and $\mathrm{Kr}$ 
a

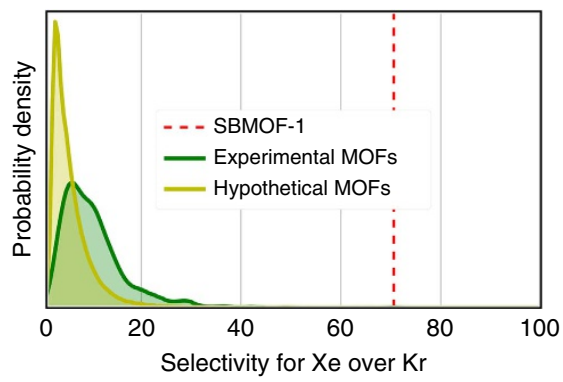

b
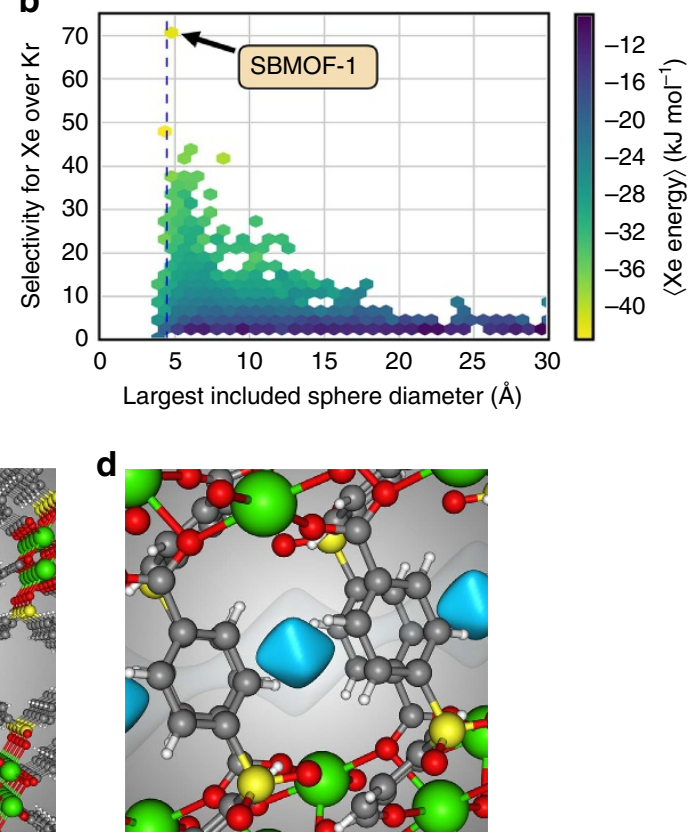

Figure 1 | Computational screening of MOFs for Xe/Kr separations at dilute conditions relevant to UNF reprocessing off-gas. We computed the Henry coefficients of $\mathrm{Xe}$ and $\mathrm{Kr}$ in $\sim 125,000$ MOF structures; the selectivity at dilute conditions is the ratio of Henry coefficients. (a) Distribution of simulated selectivities for experimentally synthesized (green) and hypothetical (yellow) MOF structures; vertical, dashed line is SBMOF-1 (KAXQIL in the Cambridge Structural Database (CSD)). (b) Histogram showing relationship between selectivity and pore size, with the largest included sphere diameter as a metric; colour shows average energy of Xe adsorption in that bin. SBMOF-1 (KAXQIL in CSD), with simulated selectivity 70.6 and largest included sphere diameter of $5.1 \AA$, is indicated. Vertical, dashed line is the distance that yields the minimum energy in a Xe-Xe Lennard-Jones potential. (c) SBMOF- 1 is composed of corner sharing, octahedrally coordinated calcium chains along the crystallographic $b$ direction, which are connected by organic linkers, forming a one-dimensional nanoporous channel. (d) Side view. Shown are the calculated potential energy contours of a Xe atom adsorbed in the pore (blue surface, $-32 \mathrm{~kJ} \mathrm{~mol}^{-1}$; white surface, $15 \mathrm{~kJ} \mathrm{~mol}^{-1}$ ).

adsorption isotherms in Co-formate ${ }^{15,20}, \mathrm{SBMOF}-2$ (ref. 13), HKUST-1 (ref. 14), MOF-505 (ref. 10), PCN-14 (ref. 19), Ni-MOF-74 (ref. 8), Zinc tetrazolate ${ }^{21}$, IRMOF-1 (ref. 22), and FMOF- $\mathrm{Cu}^{9}$ and identified the $\mathrm{Xe}$ and $\mathrm{Kr}$ Henry coefficients from the data in the low pressure regime (see Computational Calculation section of Supplementary Methods, Fig. 2b, Supplementary Figs 1-16). The saturation loading of $\mathrm{Xe}$ in SBMOF-1 is lower than observed in the majority of these materials due to the comparatively low $\left(\sim 145 \mathrm{~m}^{2} \mathrm{~g}^{-1}\right)$ surface area of SBMOF-1 (Supplementary Figs 26 and 27) ${ }^{41}$. However, the Henry coefficient of Xe in SBMOF-1 is a factor of two higher than in CC3, the material in our survey with the second highest Xe Henry coefficient; we thus expect SBMOF-1 to have an outstanding Xe uptake under UNF reprocessing off-gas conditions. Figure $2 \mathrm{~b}$ shows that SBMOF-1 exhibits by far the largest Xe Henry coefficient and the highest $\mathrm{Xe} / \mathrm{Kr}$ selectivity at dilute conditions among all reported $\mathrm{Xe}$ and $\mathrm{Kr}$ adsorption isotherms in our literature survey.

Adsorption kinetics and column breakthrough experiments. From a practical point of view, it is important that the kinetics of Xe adsorption/desorption are sufficiently fast and the material can undergo multiple ad-/de-sorption cycles without losing capacity. We measured the kinetics of Xe adsorption into an SBMOF-1 sample by connecting a chamber of Xe at 1 bar and $298 \mathrm{~K}$ to an evacuated chamber with the SBMOF-1 sample, then opening a valve to allow flow. Figure $2 \mathrm{c}$ shows that the rate of Xe uptake is sufficiently fast, reaching $\sim 80 \%$ of saturation uptake within $10 \mathrm{~min}$. Next, we performed $10 \mathrm{ad}$-/de-sorption cycles to test if SBMOF-1 retains its high $\mathrm{Xe}$ adsorption capacity after many cycles. Figure $2 \mathrm{~d}$ shows that SBMOF-1 retains its performance after multiple cycles. In addition, SBMOF-1 shows high thermal stability up to $500 \mathrm{~K}$ (Supplementary Fig. 20). To demonstrate the practical applicability of SBMOF-1 for capturing Xe from UNF reprocessing off-gas, we conducted single-column breakthrough experiments with a representative gas mixture (400 p.p.m. Xe, 40 p.p.m. $\mathrm{Kr}, 78.1 \% \mathrm{~N}_{2}, 20.9 \% \mathrm{O}_{2}, 0.03 \% \mathrm{CO}_{2}$ and $0.9 \% \mathrm{Ar}$ ) (see breakthrough measurement section of the Supplementary Information, Supplementary Figs 28 and 29) ${ }^{17}$. We fed this gas mixture through a column packed with SBMOF-1 and initially purged with He. Figure 3 shows that all gases except Xe broke through the column within minutes, whereas Xe was retained in the column for more than an hour). This demonstrates that SBMOF-1 can selectively remove Xe from air at UNF reprocessing conditions. Under these conditions, SBMOF-1 adsorbed $13.2 \mathrm{mmol}$ Xe per $\mathrm{kg}$, higher than the reported breakthrough Xe capacities of benchmark materials, Ni-MOF-74 (4.8 mmol Xe per $\mathrm{kg}$ ) and CC3 (11 mmol Xe per kg) (Supplementary Fig. 29) ${ }^{12,17}$. The experimental breakthrough capacity is close to that predicted from the Henry coefficient of the pure-component Xe isotherm $\left(15.4 \mathrm{mmol} \mathrm{kg}^{-1}\right)$, suggesting minimal diffusion limitations in the SBMOF-1 pellets. Next, we conducted column breakthrough experiments on SBMOF-1 in the presence of $42 \%$ relative humidity (Fig. 3b). Remarkably, SBMOF-1 retains a high Xe uptake $\left(\sim 11.5 \mathrm{mmol} \mathrm{kg}^{-1}\right)$ even in the presence of water vapor. These results suggest the outstanding stability of SBMOF-1 makes it a practical material for the removal of Xe from UNF reprocessing off-gas. Such stability is a desirable property, as very few metal-organic hybrid materials exhibit such properties ${ }^{42-44}$. We postulate the absence of open metal sites to be responsible for the stability of SBMOF-1 in the presence of water vapour ${ }^{45}$. 

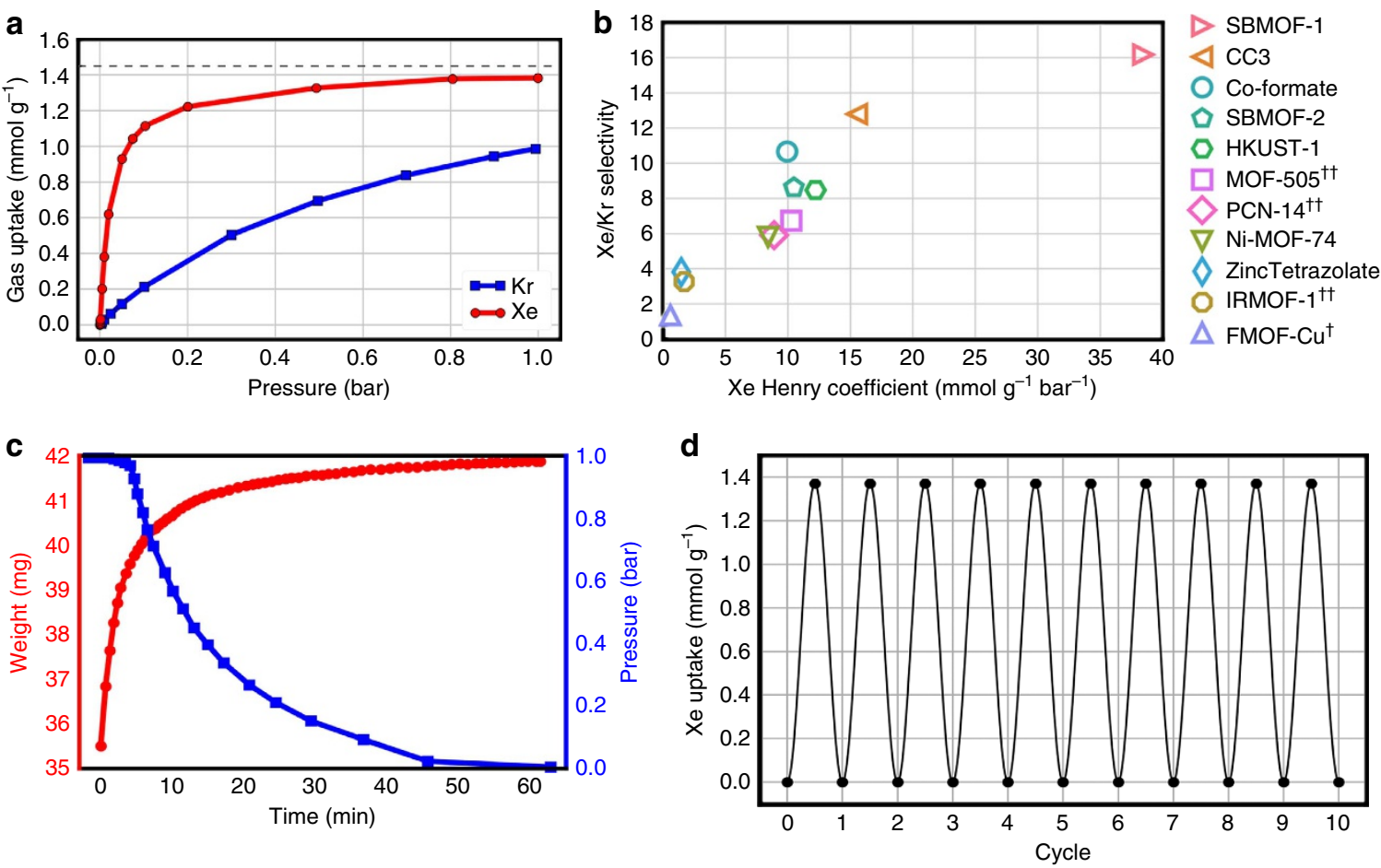

Figure 2 | Experimental characterization of Xe and Kr adsorption in SBMOF-1. (a) Experimental Xe and Kr adsorption isotherms. Horizontal line indicates one atom per pore segment. (b) Survey of thermodynamic $\mathrm{Xe} / \mathrm{Kr}$ separation performance in top-performing materials. Henry coefficients are extracted from pure-component $\mathrm{Xe}$ and $\mathrm{Kr}$ adsorption isotherms reported in the literature (see Methods). Data at $298 \mathrm{~K}$, exceptions denoted by a dagger ( $\dagger$ ) for $297 \mathrm{~K}$ and a double dagger $(\dagger \dagger)$ for $292 \mathrm{~K}$. (c) Xe adsorption kinetics experiments. The blue curve shows the pressure drop in a chamber feeding Xe to an initially evacuated chamber with the SBMOF-1 sample; the red curve shows the corresponding weight increase due to Xe adsorption. (d) Xe adsorption/ desorption cycling data; a sinusoidal curve is superimposed on the data. (a,c,d) Data for SBMOF-1 at $298 \mathrm{~K}$.
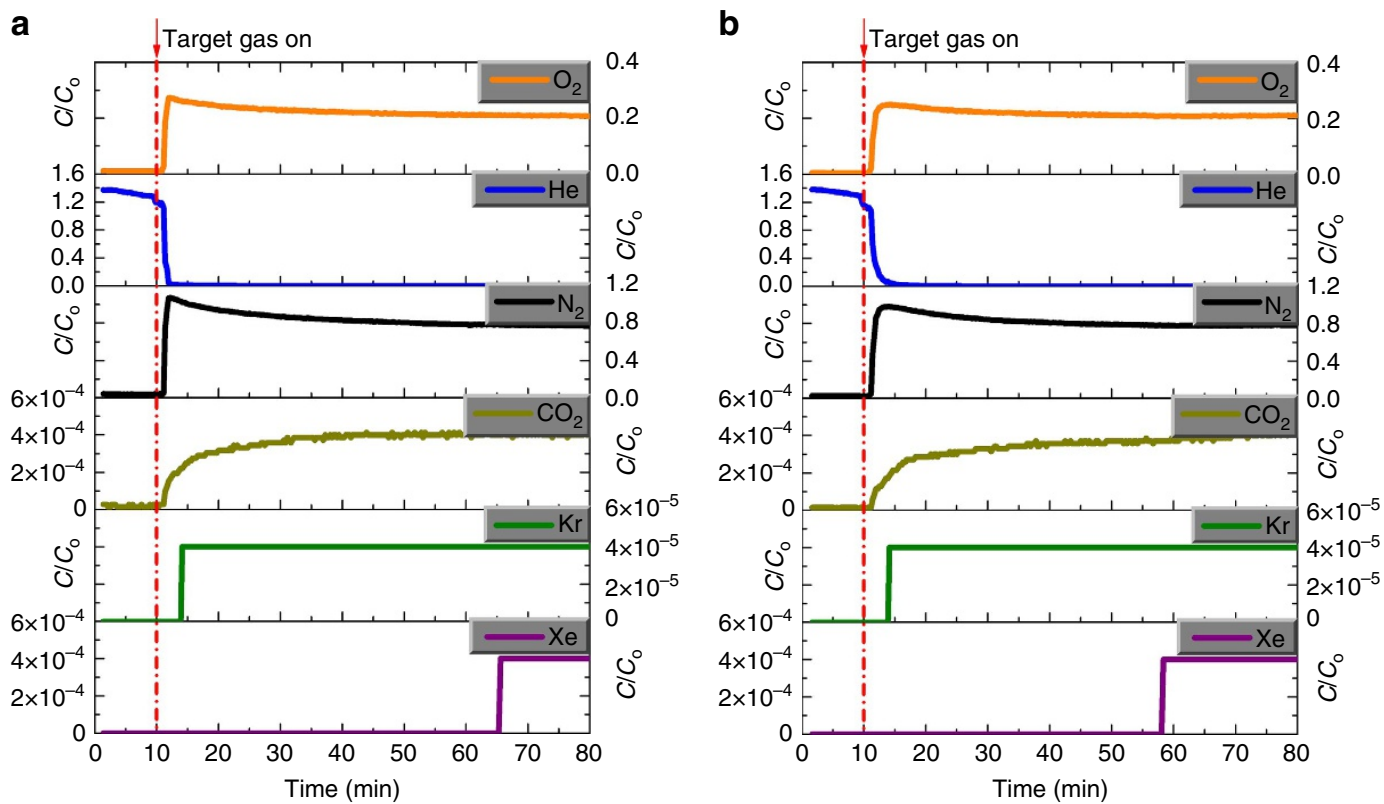

Figure 3 | Single column breakthrough experiments using SBMOF-1 at room temperature and 1 atm. Column is initially purged with He. (a) Inlet is a dry gas mixture with 400 p.p.m. Xe and 40 p.p.m. Kr balanced with air. (b) Inlet is the same gas mixture as in (a) with $42 \%$ relative humidity. Note that the Xe breakthrough time is only marginally decreased in the presence of water.

Revealing the Xe adsorption sites in SBMOF-1. To identify the location of adsorbed $\mathrm{Xe}$ and $\mathrm{Kr}$, we performed single-crystal X-ray diffraction experiments on activated SBMOF-1 (Supplementary Data 1 and 2). Single-crystal analysis of
Xe-loaded SBMOF-1 reveals that Xe adsorbs at a single site, near the midpoint of the channel, interacting with the channel wall composed of aromatic rings by mainly van der Waals interactions. Due to symmetry considerations (space group 

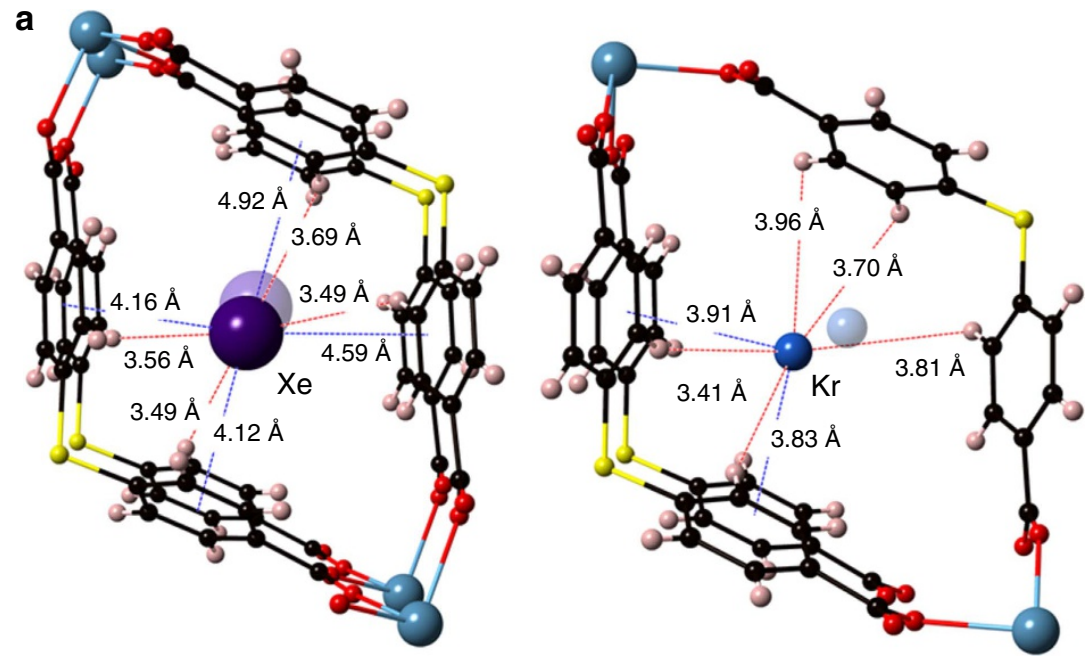

b

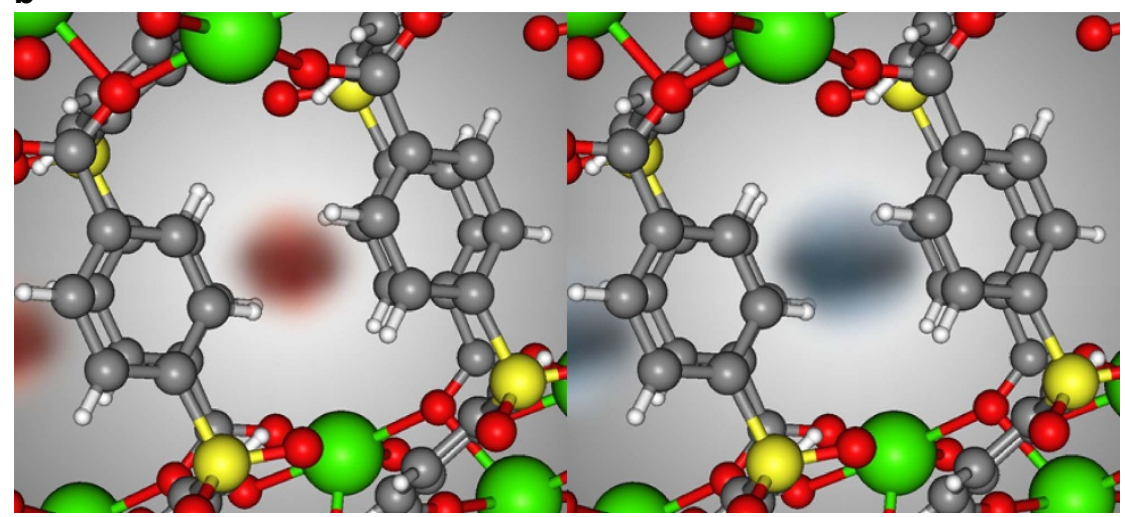

Figure 4 | Xe and Kr adsorption sites in SBMOF-1. (a) Xe and Kr positions determined by single-crystal X-ray diffraction. (b) Spatial probability densities from recording adsorbate positions during pure-component grand-canonical Monte Carlo simulations at 1 bar and $298 \mathrm{~K}$ ( $\mathrm{Xe}$, red; Kr, blue).

$P 2_{1} / \mathrm{c}$ ), each $\mathrm{Xe}$ atom is positioned at two possible sites (Fig. 4, Supplementary Table 1). The distance between each Xe atom along the $b$ axis is $5.56 \AA$, closely matching the $b$ axis length of the unit cell. There are 1.72 atoms of Xe per unit cell based on crystallographic analysis $\left(\sim 1.25 \mathrm{mmolg}^{-1}\right)$, close to the loading obtained from gas-adsorption data $\left(1.38 \mathrm{mmol} \mathrm{g}^{-1}\right)$. The saturation loading of Xe in Fig. 2a approaches two atoms per unit cell (see horizontal line), indicating commensurate Xe adsorption, which occurs when the adsorbed amount, location and orientation of an adsorbate are commensurate with the crystallographic symmetry of the adsorbent ${ }^{46}$. Such commensurate adsorption in SBMOF-1 was previously observed for small hydrocarbon molecules $\left(\mathrm{C}_{2}-\mathrm{C}_{3}\right)^{47,48}$. The observed position of $\mathrm{Xe}$ in the pore is consistent with calculated potential energy contours and molecular simulations of Xe adsorption (Figs 1d and 4b, Supplementary Fig. 30, Supplementary Table 2).

We can rationalize the high Xe adsorption capacity and selectivity exhibited by SBMOF-1 by its optimal Xe adsorption site. First, the pore size of SBMOF-1 is tailored for $\mathrm{Xe}^{35,38}$. As a metric for pore size, we calculate the diameter of the largest included hard-sphere that can fit inside the pore of SBMOF-1 as $4.2 \AA$, slightly larger than a Xe atom, $\sim 4.1 \AA$. Simulations of $\mathrm{Xe} / \mathrm{Kr}$ adsorption in the database of experimental MOFs show that all of the most selective MOFs have pore sizes slightly larger than a Xe atom (Fig. 1b). Such a pore diameter is a prerequisite for a highly Xe-selective material, as the pore size controls the proximity and degree of overlap from multiple framework atoms contributing van der Waals interactions from multiple directions to achieve a highly favourable host-Xe interaction. A pore of optimal size for $\mathrm{Xe}$ is suboptimal for $\mathrm{Kr}$ because of the size difference, so this forms a pore that is highly discriminatory for Xe over $\mathrm{Kr}^{38}$. As shown in Fig. 1c, the pore size of SBMOF-1 falls in the optimal pore size window for $\mathrm{Xe} / \mathrm{Kr}$ separations ${ }^{37}$, distinguishing it from other MOFs. Porous organic cage CC3, another outstanding Xe-selective material, also exhibits a pore size tailored for Xe (pore window $4.4 \AA$ ), but SBMOF- 1 constructs a denser wall of chemical moieties than CC3 to achieve a higher Xe binding energy, enhancing its preference for Xe (Supplementary Table 3). This is the second reason why SBMOF-1 is outstanding in Xe adsorption; the colour in Fig. 1b shows that the dense wall of SBMOF-1 surrounds a Xe atom to achieve a high energy of $\mathrm{Xe}$ adsorption and thus a high $\mathrm{Xe}$ selectivity, following the trend in other MOFs.

\section{Discussion}

We demonstrated that a nanoporous MOF, SBMOF-1, identified as an outstanding $\mathrm{Xe} / \mathrm{Kr}$ selective material from molecular simulations, shows exceptional Xe uptake at low pressure, selectivity for $\mathrm{Xe}$, thermal and water stability, and adsorption kinetics. These attributes make SBMOF-1 potentially useful as a practical, near-term material for removal of $\mathrm{Xe}$ and $\mathrm{Kr}$ from nuclear reprocessing facilities with a far less energy requirement than cryogenic distillation. The selective adsorption of Xe from relevant gas mixtures even with $\sim 42 \%$ relative humidity demonstrate practicality and offer improvements over current 
technologies. Our recent economic analysis showed the cost benefits of using Ni-MOF-74 for an adsorption-based separation process at room temperature in comparison with cryogenic distillation ${ }^{7,11}$. The discovery of the high Xe uptake and selectivity of SBMOF-1 at UNF reprocessing conditions-also in the presence of humidity-will enable an even more cost-effective process. The exceptional selectivity of SBMOF-1 is attributed to its pore size tailored to $\mathrm{Xe}$ and its dense wall of atoms that constructs a binding site with a high affinity for $\mathrm{Xe}$, as evident by single-crystal X-ray diffraction and molecular models. As molecular simulations predicted SBMOF-1 to be among the most selective of $\sim 5,000$ experimentally reported MOFs and $\sim 120,000$ hypothetical MOF structures a priori, this work is a rare case of a computationally inspired materials discovery.

\section{Methods}

Synthesis and scale up. SBMOF-1 was originally synthesized using a previously published literature procedure ${ }^{41}$. In a typical synthesis, a mixture of $0.6 \mathrm{mmol}$ of $\mathrm{CaCl}_{2}(0.074 \mathrm{~g})$ and $0.6 \mathrm{mmol}$ of $4,4^{\prime}-\mathrm{SDB}(0.198 \mathrm{~g})$ were added in $10 \mathrm{ml}$ of ethanol and stirred for $\sim 2 \mathrm{~h}$ to achieve homogeneity (molar ratio of metal chloride: ligand:solvent $=1: 1: 380$ ). The resultant solution was heated at $180^{\circ} \mathrm{C}$ for 3 days. Colourless, needle-shaped crystals were recovered as product and washed with ethanol (yield: $45 \%$ based on $\mathrm{CaCl}_{2}, 0.1 \mathrm{~g}$ ). For scale up, $1.44 \mathrm{~g}$ of $\mathrm{CaCl}_{2}(13 \mathrm{mmol})$ and $3.98 \mathrm{~g}$ of $4,4^{\prime}$-SDB $(13 \mathrm{mmol})$ were added to $120 \mathrm{ml}$ of ethanol and stirred for $\sim 2 \mathrm{~h}$ to achieve homogeneity (molar ratio: 1:1:156). The well-mixed solution was then transferred to three $100 \mathrm{ml}$ Teflon-lined stainless steel Parr autoclaves and heated for 3 days at $180^{\circ} \mathrm{C}$. The product was obtained as white powder and washed by ethanol ( 3 times, $50 \mathrm{ml}$ ), followed by drying under vacuum (yield: $2.2 \mathrm{~g}, 50 \%$ based on $\mathrm{CaCl}_{2}$ ). The as-synthesized material was then exchanged with methanol $(3 \times, 50 \mathrm{ml})$ for a total period of 3 days. The product purity was confirmed by powder XRD.

Gas-adsorption and breakthrough experiments. The methanol-exchanged SBMOF-1 was activated at $100^{\circ} \mathrm{C}$ for $12 \mathrm{~h}$ under dynamic vacuum. Singlecomponent gas-adsorption isotherms were collected in a Quantachrome Autosorb1 and dynamic sorption analyzer (ARBC, Hiden Analytical Ltd., Warrington, UK). The later instrument was also used to collect breakthrough measurement data. Breakthrough measurements were conducted on $20-35$ mesh $(500-850 \mu \mathrm{m})$ pellets of SBMOF-1 (1.48g) using a gas mixture composition simulating UNF conditions (400 p.p.m. Xe, 40 p.p.m. Kr, $78.1 \% \mathrm{~N}_{2}, 20.9 \% \mathrm{O}_{2}, 0.03 \% \mathrm{CO}_{2}$ and $0.9 \% \mathrm{Ar}$ ).

Computational methodologies. At dilute conditions relevant to UNF reprocessing off-gas, we modelled Xe and Kr adsorption in the MOFs with Henry's law. Let $P$ be the pressure (units: bar) and $\sigma(P)$ be the gas uptake (units: $\mathrm{mmol} \mathrm{g}^{-1}$ ) as a function of pressure (the adsorption isotherm). Henry's law, only valid at low surface coverage, is then:

$$
\sigma(P)=K_{\mathrm{H}} P,
$$

where $K_{\mathrm{H}}$ is the Henry coefficient (units: $\mathrm{mmolg}^{-1} \mathrm{bar}^{-1}$ ) of the gas in the adsorbent. The $\mathrm{Xe} / \mathrm{Kr}$ selectivity is then the ratio of the Henry coefficients. We calculated the Henry coefficient in each MOF using Widom particle insertions, a Monte Carlo integration ${ }^{49}$. We model the energetic interactions between Xe and $\mathrm{Kr}$ with the atoms of the MOFs using Lennard Jones potentials. We took parameters for $\mathrm{Xe}$ and $\mathrm{Kr}$ from Boato et al. and for the MOF atoms from the Universal Force Field, applying Lorentz-Berthelot mixing rules to obtain cross-interactions ${ }^{50,51}$. We hold the MOF structures rigid throughout the simulation and apply periodic boundary conditions to mimic an infinite crystal. For potential energy contours and spatial probability density plots for SBMOF-1, we utilized a hybrid DreidingTraPPE force field, as this force field produces a better match to the Xe and $\mathrm{Kr}$ isotherms in SBMOF-1 than the UFF ${ }^{52,53}$. The largest included hard sphere diameter is calculated using Zeo ++ (refs 54,55). See Supplementary Methods for more details.

Literature survey for pure-component $\mathrm{Xe}$ and $\mathbf{K r}$ adsorption isotherms. To generate Fig. 2b, we collected from the literature experimentally measured singlecomponent $\mathrm{Xe}$ and $\mathrm{Kr}$ adsorption isotherms in MOFs and porous organic cage materials. Focusing on the low-pressure regime of the adsorption isotherm that exhibits linear behaviour-the Henry regime where Henry's law in equation (1) is valid-we fit a line with zero intercept to this data to identify $K_{\mathrm{H}}$ of Xe and $\mathrm{Kr}$ in the material. See Supplementary Section for the data and visualizations of the resulting fits to equation (1). Our data and code to reproduce Fig. $2 \mathrm{~b}$ are openly available on GitHub at https://github.com/CorySimon/XeKrMOFAdsorptionSurvey.

Single-crystal X-ray diffraction. The single-crystal data on the Xe- and Kr-loaded activated SBMOF-1 were collected using a four circle kappa Oxford Gemini diffractometre equipped with an Atlas detector $(\lambda=0.71073)$ at $100 \mathrm{~K}$. The raw intensity data were collected, integrated and corrected for absorption effects using CrysAlis PRO software. Data sets were corrected for absorption using a multi-scan method, and structures were solved by direct methods using SHELXS-97 and refined by full-matrix least squares on $F^{2}$ with SHELXL-97 (ref. 56).

Data availability. The X-ray crystallographic coordinates for structures reported in this study have been deposited at the Cambridge Crystallographic Data Centre (CCDC), under deposition numbers 1475229-1475230. These data can be obtained free of charge from the Cambridge Crystallographic Data Centre via www.ccdc.cam.ac.uk/data_request/cif. Our data and code to reproduce Fig. $2 b$ are openly available on GitHub at https://github.com/CorySimon/XeKrMOFAdsorptionSurvey. All other data, if not included in the Article or the Supplementary Information, are available from the authors on request.

\section{References}

1. Hoffert, M. I. et al. Advanced technology paths to global climate stability: energy for a greenhouse planet. Science 298, 981-987 (2002).

2. Chu, S. \& Majumdar, A. Opportunities and challenges for a sustainable energy future. Nature 488, 294-303 (2012).

3. Lenzen, M. Life cycle energy and greenhouse gas emissions of nuclear energy: a review. Energ. Convers. Manage. 49, 2178-2199 (2008).

4. Soelberg, N. R. et al. Radioactive iodine and krypton control for nuclear fuel reprocessing facilities. Sci. Technol. Nucl. Ins. 2013, 1-12 (2013).

5. Ying, R. T. Gas Separation by Adsorption Processes (Butterworth-Heinemann, 2013).

6. Kerry, F. G. Industrial Gas Handbook: Gas Separation and Purification (CRC Press, 2007).

7. Liu, J., Fernandez, C. A., Martin, P. F., Thallapally, P. K. \& Strachan, D. M. A two-column method for the separation of $\mathrm{Kr}$ and Xe from process off-gases. Ind. Eng. Chem. Res. 53, 12893-12899 (2014).

8. Thallapally, P. K., Grate, J. W. \& Motkuri, R. K. Facile xenon capture and release at room temperature using a metal-organic framework: a comparison with activated charcoal. Chem. Commun. (Camb) 48, 347-349 (2012).

9. Fernandez, C. A., Liu, J., Thallapally, P. K. \& Strachan, D. M. Switching Kr/Xe selectivity with temperature in a metal-organic framework. J. Am. Chem. Soc. 134, 9046-9049 (2012).

10. Bae, Y. S. et al. High xenon/krypton selectivity in a metal-organic framework with small pores and strong adsorption sites. Micropor. Mesopor. Mater. 169, 176-179 (2013).

11. Banerjee, D. et al. Potential of metal-organic frameworks for separation of xenon and krypton. Acc. Chem. Res. 48, 211-219 (2014).

12. Chen, L. et al. Separation of rare gases and chiral molecules by selective binding in porous organic cages. Nat. Mater. 13, 954-960 (2014).

13. Chen, X. et al. Direct observation of Xe and $\mathrm{Kr}$ adsorption in a Xe-selective microporous metal-organic framework. J. Am. Chem. Soc. 137, 7007-7010 (2015).

14. Hulvey, Z. et al. Noble gas adsorption in copper trimesate, HKUST-1: an experimental and computational study. J. Phys. Chem. C 117, 20116-20126 (2013).

15. Lawler, K. V., Hulvey, Z. \& Forster, P. M. Nanoporous metal formates for krypton/xenon separation. Chem. Commun. (Camb) 49, 10959-10961 (2013).

16. Liu, J., Strachan, D. M. \& Thallapally, P. K. Enhanced noble gas adsorption in Ag@MOF-74Ni. Chem. Commun. (Camb) 50, 466-468 (2014).

17. Liu, J., Thallapally, P. K. \& Strachan, D. Metal-organic frameworks for removal of Xe and $\mathrm{Kr}$ from nuclear fuel reprocessing plants. Langmuir 28, 11584-11589 (2012).

18. Meek, S. T., Teich-McGoldrick, S. L., Perry, J. J., Greathouse, J. A. \& Allendorf, M. D. Effects of polarizability on the adsorption of noble gases at low pressures in monohalogenated isoreticular metal-organic frameworks. J. Phys. Chem. C 116, 19765-19772 (2012)

19. Perry, J. J. et al. Noble gas adsorption in metal-organic frameworks containing open metal sites. J. Phys. Chem. C 118, 11685-11698 (2014).

20. Wang, H. et al. The first example of commensurate adsorption of atomic gas in a MOF and effective separation of xenon from other noble gases. Chem. Sci. 5, 620-624 (2014).

21. Xiong, S. S. et al. A flexible zinc tetrazolate framework exhibiting breathing behaviour on xenon adsorption and selective adsorption of xenon over other noble gases. J. Mater. Chem. A 3, 10747-10752 (2015).

22. Mueller, U. et al. Metal-organic frameworks - prospective industrial applications. J. Mater. Chem. 16, 626-636 (2006).

23. Yaghi, O. M. et al. Reticular synthesis and the design of new materials. Nature 423, 705-714 (2003).

24. Zhou, H.-C., Long, J. R. \& Yaghi, O. M. Introduction to metal-organic frameworks. Chem. Rev. 112, 673-674 (2012). 
25. Eddaoudi, M., Sava, D. F., Eubank, J. F., Adil, K. \& Guillerm, V. Zeolite-like metal-organic frameworks (ZMOFs): design, synthesis, and properties. Chem. Soc. Rev. 44, 228-249 (2015).

26. Ferey, G. Hybrid porous solids: past, present, future. Chem. Soc. Rev. 37, 191-214 (2008)

27. James, S. L. Metal-organic frameworks. Chem. Soc. Rev. 32, 276-288 (2003).

28. Kitagawa, S., Kitaura, R. \& Noro, S.-i. Functional porous coordination polymers. Angew. Chem. Int. Ed. 43, 2334-2375 (2004).

29. Li, J.-R., Kuppler, R. J. \& Zhou, H.-C. Selective gas adsorption and separation in metal-organic frameworks. Chem. Soc. Rev. 38, 1477-1504 (2009).

30. Sumida, K. et al. Carbon dioxide capture in metal-organic frameworks. Chem. Rev. 112, 724-781 (2012).

31. Lee, J. et al. Metal-organic framework materials as catalysts. Chem. Soc. Rev. 38, 1450-1459 (2009).

32. Kreno, L. E. et al. Metal-organic framework materials as chemical sensors. Chem. Rev. 112, 1105-1125 (2012).

33. Allendorf, M. D., Bauer, C. A., Bhakta, R. K. \& Houk, R. J. T. Luminescent metal-organic frameworks. Chem. Soc. Rev. 38, 1330-1352 (2009).

34. Motkuri, R. K. et al. Fluorocarbon adsorption in hierarchical porous frameworks. Nat. Commun. 5, 4368 (2014).

35. Ryan, P., Farha, O. K., Broadbelt, L. J. \& Snurr, R. Q. Computational screening of metal-organic frameworks for xenon/krypton separation. Aiche J. 57, 1759-1766 (2011).

36. Van Heest, T., Teich-McGoldrick, S. L., Greathouse, J. A., Allendorf, M. D. \& Sholl, D. S. Identification of metal-organic framework materials for adsorption separation of rare gases: applicability of ideal adsorbed solution theory (IAST) and effects of inaccessible framework regions. J. Phys. Chem. C 116, 13183-13195 (2012).

37. Sikora, B. J., Wilmer, C. E., Greenfield, M. L. \& Snurr, R. Q. Thermodynamic analysis of $\mathrm{Xe} / \mathrm{Kr}$ selectivity in over 137000 hypothetical metal-organic frameworks. Chem. Sci. 3, 2217 (2012).

38. Simon, C. M., Mercado, R., Schnell, S. K., Smit, B. \& Haranczyk, M. What are the best materials to separate a xenon/krypton mixture? Chem. Mater. 27, 4459-4475 (2015).

39. Chung, Y. G. et al. Computation-ready, experimental metal-organic frameworks: a tool to enable high-throughput screening of nanoporous crystals. Chem. Mater. 26, 6185-6192 (2014).

40. Wilmer, C. E. et al. Large-scale screening of hypothetical metal-organic frameworks. Nat. Chem. 4, 83-89 (2012).

41. Banerjee, D., Zhang, Z. J., Plonka, A. M., Li, J. \& Parise, J. B. A calcium coordination framework having permanent porosity and high $\mathrm{CO} 2 / \mathrm{N}-2$ selectivity. Cryst Growth Des 12, 2162-2165 (2012).

42. Howarth, A. J. et al. Chemical, thermal and mechanical stabilities of metal-organic frameworks. Nat. Rev. Mater. 1, 15018 (2016).

43. Deria, P. et al. Ultraporous, water stable, and breathing zirconium-based metal-organic frameworks with ftw topology. J. Am. Chem. Soc. 137, 13183-13190 (2015).

44. Kalidindi, S. B. et al. Chemical and structural stability of zirconium-based metal-organic frameworks with large three-dimensional pores by linker engineering. Angew. Chem. Int. Ed. Engl. 127, 223-228 (2015).

45. Kizzie, A. C., Wong-Foy, A. G. \& Matzger, A. J. Effect of humidity on the performance of microporous coordination polymers as adsorbents for $\mathrm{CO} 2$ Capture. Langmuir 27, 6368-6373 (2011).

46. Wu, H., Gong, Q., Olson, D. H. \& Li, J. Commensurate adsorption of hydrocarbons and alcohols in microporous metal organic frameworks. Chem. Rev. 112, 836-868 (2012).

47. Banerjee, D. et al. Direct structural evidence of commensurate-toincommensurate transition of hydrocarbon adsorption in a microporous metal organic framework. Chem. Sci. 7, 759-765 (2016).

48. Plonka, A. M. et al. Light hydrocarbon adsorption mechanisms in two calciumbased microporous metal organic frameworks. Chem. Mater. 28, 1636-1646 (2016).

49. Frenkel, D. \& Smit, B. Understanding Molecular Simulation: From Algorithms to Applications (Academic Press, 2001).

50. Boato, G. \& Casanova, G. A self-consistent set of molecular parameters for neon, argon, krypton and xenon. Physica 27, 571 -\& (1961).
51. Rappe, A. K., Casewit, C. J., Colwell, K. S., Goddard, W. A. \& Skiff, W. M. Uff a full periodic-table force-field for molecular mechanics and molecular-dynamics simulations. J. Am. Chem. Soc. 114, 10024-10035 (1992).

52. Wick, C. D., Martin, M. G. \& Siepmann, J. I. Transferable potentials for phase equilibria. 4. United-atom description of linear and branched alkenes and alkylbenzenes. J. Phys. Chem. B 104, 8008-8016 (2000).

53. Mayo, S. L., Olafson, B. D. \& Goddard, W. A. Dreiding - a generic force-field for molecular simulations. J. Phys. Chem. 94, 8897-8909 (1990).

54. Willems, T. F., Rycroft, C., Kazi, M., Meza, J. C. \& Haranczyk, M. Algorithms and tools for high-throughput geometry-based analysis of crystalline porous materials. Micropor. Mesopor. Mater. 149, 134-141 (2012).

55. Pinheiro, M., Martin, R. L., Rycroft, C. H. \& Haranczyk, M. High accuracy geometric analysis of crystalline porous materials. CrystEngComm 15, 7531-7538 (2013).

56. Sheldrick, G. M. A short history of SHELX. Acta Cryst. A. 64, 112-122 (2008)

\section{Acknowledgements}

We (PNNL) acknowledge US Department of Energy (DOE), Office of Nuclear Energy for synthesis, $\mathrm{Xe} / \mathrm{Kr}$ adsorption, kinetics and breakthrough measurements. C.M.S. is supported by the US DOE, Office of Science, Office of Workforce Development for Teacher and Scientists, Office of Science Graduate Student Research (SCGSR) program. The SCGSR program is administered by the Oak Ridge Institute for Science and Education for the DOE under Contract No. DE-AC05-06OR23100. B.S. is supported by the Center for Gas Separations Relevant to Clean Energy Technologies, an Energy Frontier Research Center funded by the US DOE, Office of Science, Office of Basic Energy Sciences under Award No. DE-SC0001015. M.H. was supported by the Center for Applied Mathematics for Energy Research Applications (CAMERA), funded by the U.S. Department of Energy under Contract No. DE-AC02- 05CH11231. This research used resources of the National Energy Research Scientific Computing Center, which is supported by the Office of Science of the US DOE under Contract No. DE-AC02-05CH11231. A.M.P., X.C. and J.B.P. were supported by the National Science Foundation DMR-1231586 and CHE-0840483. P.K.T. would like to acknowledge Dr Terry Todd at Idaho National Laboratory, Dr Robert Jubin at Oakridge National Laboratory, Dr Denis Strachan, Dr John Vienna at PNNL, Kimberly Gray (DOE-NE HQ) and Jim Breese (DOE-NE HQ) for programmatic support. PNNL is a multi-program national laboratory operated for the US DOE by Battelle Memorial Institute under Contract DE-AC05-76RL01830.

\section{Author contributions}

D.B. synthesized SBMOF-1, and performed scale-up operation, activation and single-component gas-adsorption experiments. J.L. and D.B. performed breakthrough measurement. R.K.M. performed cycle studies. C.M.S., B.S. and M.H. performed computational work. A.M.P., X.C. and J.B.P. performed single-crystal X-ray diffraction studies. P.K.T. conceived and executed the project. P.K.T., D.B., C.M.S., B.S. and M.H. wrote the manuscript with input from all the co-authors.

\section{Additional information}

Supplementary Information accompanies this paper at http://www.nature.com/ naturecommunications

Competing financial interests: The authors declare no competing financial interests.

Reprints and permission information is available online at http://npg.nature.com/ reprintsandpermissions/

How to cite this article: Banerjee, D. et al. Metal-organic framework with optimally selective xenon adsorption and separation. Nat. Commun. 7:11831 doi: 10.1038/ ncomms11831 (2016).

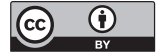

This work is licensed under a Creative Commons Attribution 4.0 International License. The images or other third party material in this article are included in the article's Creative Commons license, unless indicated otherwise in the credit line; if the material is not included under the Creative Commons license, users will need to obtain permission from the license holder to reproduce the material. To view a copy of this license, visit http://creativecommons.org/licenses/by/4.0/ 